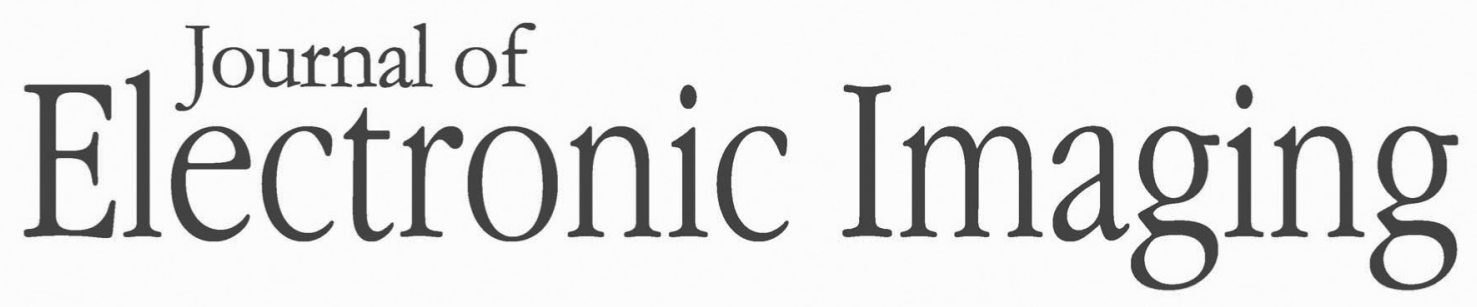

SPIEDigitalLibrary.org/jei

\title{
Sparse ground-penetrating radar imaging method for off-the-grid target problem
}
Ali Cafer Gurbuz
Oguzhan Teke
Orhan Arikan

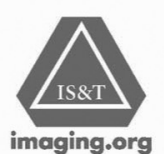




\title{
Sparse ground-penetrating radar imaging method for off-the-grid target problem
}

\author{
Ali Cafer Gurbuz \\ TOBB University of Economics and Technology \\ Department of Electrical and Electronics Engineering \\ Sogutozu Cad. No 43, Ankara 06560, Turkey \\ E-mail: acgurbuz@etu.edu.tr \\ Oguzhan Teke \\ Orhan Arikan \\ Bilkent University \\ Department of Electrical and Electronics Engineering \\ TR-06800 Bilkent, Ankara, Turkey
}

\begin{abstract}
Spatial sparsity of the target space in subsurface or through-the-wall imaging applications has been successfully used within the compressive-sensing framework to decrease the data acquisition load in practical systems, while also generating highresolution images. The developed techniques in this area mainly discretize the continuous target space into grid points and generate a dictionary of model data that is used in image-reconstructing optimization problems. However, for targets that do not coincide with the computation grid, imaging performance degrades considerably. This phenomenon is known as the off-grid problem. This paper presents a novel sparse ground-penetrating radar imaging method that is robust for off-grid targets. The proposed technique is an iterative orthogonal matching pursuit-based method that uses gradientbased steepest ascent-type iterations to locate the off-grid target. Simulations show that robust results with much smaller reconstruction errors are obtained for multiple off-grid targets compared to standard sparse reconstruction techniques. (C) 2013 SPIE and IS\&T [DOI: 10 .1117/1.JEI.22.2.021007]
\end{abstract}

\section{Introduction}

Ground-penetrating radar (GPR) has been widely used in detecting or imaging subsurface targets. ${ }^{1,2}$ Traditional GPRs image the subsurface by transmitting short electromagnetic (EM) pulses or a train of sub-pulses with stepped carrier frequencies and processing the reflections caused by permittivity discontinuities in the ground. Imaging with GPR requires the formation of a synthetic aperture, which is done by scanning a GPR sensor over the region of interest and recording the time/frequency signal returns for many spatial positions. This operation generates a large amount of space-time/frequency data. State-of-the-art GPR systems operate with large bandwidths and a large number of channels, which further increases the amount of data for processing. However, in most GPR applications, the target space consists of a small number of strong targets. For example,

Paper 12317SS received Aug. 17, 2012; revised manuscript received Nov. 8, 2012; accepted for publication Nov. 19, 2012; published online Jan. 31, 2013.

0091-3286/2013/\$25.00 @ 2013 SPIE and IS\&T potential targets, such as buried landmines or metallic objects, cover a small part of the total subsurface to be imaged. Hence, a large amount of data is processed to detect or image a small number of targets. This provides the opportunity to apply sparse signal-processing techniques for GPR applications.

Recent results in the theory of compressive sensing $(\mathrm{CS})^{3-7}$ show that reconstruction of unknown signals, which have a sparse representation in a certain transformation domain, can be obtained from a much smaller set of measurements compared to conventional techniques. Because GPR applications may admit a sparse representation, reconstruction of target scenes can be formulated as a sparse signal-reconstruction problem. Based on this main idea, application of CS to GPR imaging has been investigated in several works. In one of the earliest papers on CS applied to the general context of radar, ${ }^{8}$ initial ideas about the possibility of subNyquist sampling and elimination of match filtering in radar was discussed. Application of CS to a GPR imaging problem was first demonstrated in Ref. 9. In that work, the subsurface area was modeled to consist of a small number of discrete point-like targets, and a dictionary of model data was generated for each possible discrete target point. The subsurface image was generated by solving an $l_{1}$ minimizationbased optimization problem with a decreased number of measurements. Later, these results were extended to the stepped frequency ${ }^{10}$ and impulse GPR ${ }^{11}$ cases. In Ref. 12, Yoon and Amin used CS for through-the-wall imaging (TWI) using wide-band beamforming, where the unmeasured frequency points are reconstructed with CS and conventional wideband beamforming is applied on the reconstructed measurements. In Ref. 13, CS was applied to stepped-frequency GPRs. Sparse signal reconstruction ideas are applied to GPR rebar detection in Ref. 14. CS for TWI are further explored in Refs. 15 and 16.

All the above CS-GPR imaging methods mainly discretize the continuous target space, generate a number of grid points, and assume that the targets are exactly positioned on the center of these grids. Furthermore, medium parameters such as permittivity or wave velocities are assumed to be 
perfectly known. These assumptions were used to build a forward model between the discretized target space and measurements, resulting in a dictionary of data formed by synthesizing the model GPR data for each discrete target space position. If the targets are located exactly on the grid points, the sparsity requirement of CS is satisfied and CS theory works. However, in general, a target might not be exactly on the grid center. Depending on the discretization density, actual points will be located off the grid at varying distances. One simplistic approach is to use multiresolution refinement strategy and decrease grid sizes iteratively. However, using a smaller grid size is not a direct solution to the target offthe-grid problem. First, the coherence between dictionary columns is increased, making restricted isometry property $(\mathrm{RIP})^{17}$ invalid; second, computational complexity of reconstruction is increased due to the greater size of the optimization problem. It has been discussed in both GPR and radar literature that off-grid targets cause an important degradation in CS reconstruction performance. ${ }^{18,19}$ In the Refs. 20-22, the effect of general basis mismatch, which is a direct consequence of off-the-grid targets on the reconstruction performance of CS, is analyzed and the resultant performance degradation levels and analytical $l_{2}$ norm error bounds are shown. In CS for noiseless case recovery, the error bound for a general signal is $\left\|x^{*}-x\right\|_{1} \leq$ $\left\|x-x_{k}\right\|_{1}$, but on the other hand, it is shown for a perturbed signal in Ref. 21 that for $\Psi=I+E$ and $x=\Psi \theta$ with $\theta$ being a sparse or compressible signal and no noise in the system, if the rows $e_{m} \in C^{1 x N} m=1,2, \ldots, N$ of $E$ are bounded as $\left\|e_{m}\right\|_{2}<\beta$ for $1 \leq m \leq N$, then

$$
\left\|x-x_{k}\right\|_{1} \leq(N-k) \beta\|\theta\|_{2}+\left\|\theta-\theta_{k}\right\|_{1} .
$$

This shows that the error bound for the perturbed case is larger than the classic CS bound for the unperturbed case by an amount of $(N-k) \beta\|\theta\|_{2}$. If the signal is exactly $k$ sparse, then $\left\|\theta-\theta_{k}\right\|_{1}$ is zero, which shows that the upper bound on the error norm is linearly increasing in $N, \beta$, and $\|\theta\|_{2}$ and decreasing with $k$. These papers have shown effect of mismatch on several cases such as reconstructing signals in discrete Fourier transform domain and concluded that performance of compressed sensing for approximating a sparse physical field may degrade considerably in the presence of basis mismatch, even when the assumed basis corresponds to a fine-grained discretization of the parameter space. However, in these works, a systematic approach on how to do the sparse reconstruction and imaging under the offgrid problem has not been discussed.

In this paper, a new iterative sparse reconstruction method that can also handle off-grid targets is presented. The proposed method uses the orthogonal matching pursuit $(\mathrm{OMP})^{23}$ framework for reconstruction. In the OMP algorithm, the dictionary column that has the largest correlation with the current residual is selected, and the new residual is calculated by projecting the measurements to the subspace defined by the span of all selected columns. This procedure is repeated until the termination criterion is met. OMP is a much-used reconstruction technique for radar imaging problems, mainly owing to its relatively low computational complexity and high reconstruction performance. For GPR imaging it is shown in Ref. 24 that the performance of OMP reconstruction is similar to that of $l_{1}$ minimization, but with much lower reconstruction times.
In the proposed technique, for each added grid point, steepest-descent type iterations are run to find the off-grid target within that grid. Here, the idea is to find the position that maximizes the cost function of OMP. This is achieved by iteratively calculating gradients on each spatial axis at the current position, starting from the grid center, and taking controlled steps in the directions of the gradients. These iterations can be terminated if the calculated gradients are smaller then a determined threshold. In the proposed method, the model data corresponding to the final off-grid points are generated, and the residual is calculated by projecting the measurements to the span of these model data. This procedure provides a better fit to the measurements by decreasing the modeling error due to off-grid targets.

Simulation results show that the proposed method is successful in recovering a sparse GPR image independent of whether the targets are on the grid. Whereas standard sparse reconstruction techniques create nonsparse images due to off-grid targets, the proposed method is shown to generate images with correct sparsity levels. The proposed method is compared to standard OMP for performance comparisons of the methods under varying noise levels and measurement number. It is observed that lower reconstruction errors are obtained for all performance tests. In addition to solving the off-grid problem, the proposed method is also simple to implement and faster in comparison to $l_{1}$ minimizationbased optimizations. However, it is also computationally more complex compared to OMP owing to the additional gradient and model data calculations within the OMP framework.

The organization of the paper is as follows. Section 2 outlines the CS-based GPR imaging formulation and the sparse GPR-imaging framework. Derivation of the proposed method is done in Sec. 3. Simulation results and performance comparisons are given in Sec. 4. Conclusions are drawn in Sec. 5.

\section{Compressive Sensing-Based GPR Imaging}

GPR systems transmit short EM pulses or a train of subpulses with stepped carrier frequencies at each spatial position, forming a synthetic array during the data acquisition process. The total subsurface response, which is a superposition of the responses from all reflectors within the medium, can be inverted using various techniques. There are excellent references on GPR data processing, including Refs. 1, 2, and 25. For the purposes of this paper, an example GPR scenario will be used to illustrate the proposed technique. However, this framework can be extended to other types of GPR applications with small modifications. In our model, we consider a subsurface imaging scenario with a two-layer model, where the GPR antennas will be in air and the targets are in a homogeneous medium with known permittivity level. A stepped-frequency GPR (SF-GPR) is considered, as it is more suitable for a compressive-sensing data acquisition model. As the SF-GPR scans a region, at each scan position it transmits $L$ continuous sinusoidal signals, sequentially changing the frequency. The transmitted signal for frequency $l, \omega_{\ell}=2 \pi\left(f_{0}+\ell \Delta f\right)$, can be written as

$$
E_{T}(\ell, t)=A e^{-j 2 \pi\left(f_{0}+\ell \Delta f\right) t}=A e^{-j \omega_{\ell} t},
$$


where $l=0,1,2, \ldots, L-1$, with $L$ being the total number of frequency steps, $\Delta f$ the frequency-step interval, and $f_{0}$ the initial frequency. $A$ is the strength of the transmitted signal.

Assuming $K$ dominant point targets, the received frequency measurements at the $i$ th scan point $y_{i}$ can be expressed as

$$
\boldsymbol{y}_{i}(\ell)=\sum_{k=1}^{K} \alpha_{k} e^{-j \omega_{\ell}\left[t-\tau_{i}\left(\boldsymbol{p}_{k}\right)\right]},
$$

where $\tau_{i}\left(\boldsymbol{p}_{k}\right)$ is the time delay for the target at position $\boldsymbol{p}_{k}=$ $\left[x_{k} ; y_{k} ; z_{k}\right]$ when the GPR antenna is at the $i$ th scan point, and $\alpha_{k}$ is the weight parameter for target $k$ including target reflectivity and all propagation effects. At this point, the conventional practice is to employ a matched filter of the measured data with the impulse response of the GPR data acquisition process. Diverging from the conventional methods, CS-based imaging first discretizes the target space and tries to generate a linear relation between the measured data and the target space to be imaged. This is accomplished by generating a data dictionary. For this purpose, the target space $\boldsymbol{\pi}_{T}$ which lies in the product space $\left[x_{o}, x_{f}\right] \times\left[y_{o}, y_{f}\right] \times\left[z_{o}, z_{f}\right]$ must be discretized. Here $\left(x_{o}, y_{o}, z_{o}\right)$ and $\left(x_{f}, y_{f}, z_{f}\right)$ denote the initial and final positions of the target space to be imaged along each axis. Discretization generates a finite set of $N$ target points $\mathcal{B}=\left\{\boldsymbol{\pi}_{1}, \boldsymbol{\pi}_{2}, \ldots, \boldsymbol{\pi}_{N}\right\}$, where $N$ determines the grid resolution and each $\pi_{n}$ is a 3 -D vector $\left[x_{n} ; y_{n} ; z_{n}\right]$. For any target at grid point $\boldsymbol{\pi}_{n}$, the received frequency data can be modeled using Eq. (3) with $\alpha_{k}=1$ for $K=1$. A data dictionary for the $i$ 'th GPR scan location can be generated by synthesizing the time-frequency data for each possible target space point $\pi_{n} n=1,2, \ldots, N$. Hence, the $n$th column of the data dictionary $\boldsymbol{\Psi}_{i}$ which corresponds to a target at $\boldsymbol{\pi}_{n}$, will be

$$
\left[\boldsymbol{\Psi}_{i}\right]_{n}=\exp \left\{-j \omega\left[t-\tau_{i}\left(\boldsymbol{\pi}_{n}\right)\right]\right\}
$$

where $\omega$ is the vector of $L$ frequencies. To have uniform column norms, each dictionary column can be normalized to have norm one. Note that $\boldsymbol{\Psi}_{i}$ is the dictionary for only the $i$ 'th scan position, and its dimension will be $L \times N$. If all the $K$ targets fall exactly on the grid centers, then the measurements at the $i$ 'th scan point $\boldsymbol{y}_{i}$ can be represented as a linear combination of the dictionary columns of $\boldsymbol{\Psi}_{i}$ as $\boldsymbol{y}_{i}=\boldsymbol{\Psi}_{i} \boldsymbol{b}$, where $\boldsymbol{b}$ is a vector defining the discrete target space, i.e., if there is a target at $\boldsymbol{\pi}_{n}$, then the $n$ 'th index of $\boldsymbol{b}$ will be $\alpha_{n}$, otherwise zero. Hence, if all $K$ targets are on the grid, then the vector $\boldsymbol{b}$ should be a $K$ sparse vector. However, actual targets will not be exactly on the grid centers, and since their response is not modeled in the dictionary, an approximate relationship

$$
\boldsymbol{y}_{i}=\boldsymbol{\Psi}_{i} \boldsymbol{b}+\boldsymbol{n}
$$

is used, where the term $\boldsymbol{n}$ compensates for any error due to discretization of the target space, any modeling errors or unmodeled factors, and the true measurement noise of the system. The goal is to find a sparse $\boldsymbol{b}$, which is actually an image of the medium using the measurements $\boldsymbol{y}_{i}$ at all scan points $i=1,2 \ldots S$, where $S$ represents the total number of spatial measurements. CS theory requires a much smaller number of random measurements to reconstruct a sparsely represented signal. Hence, instead of measuring all the $L$ frequency step, a random subset of $M$ frequencies for each scan position can be measured. This can be stated in matrix form as

$$
\beta_{i}=\boldsymbol{\Phi}_{i} \boldsymbol{y}_{i}=\boldsymbol{\Phi}_{i} \boldsymbol{\Psi}_{i} \boldsymbol{b}+\tilde{\boldsymbol{n}},
$$

where $\boldsymbol{\Phi}_{i}$ is an $M \times L$ measurement matrix constructed by randomly selecting $M$ rows of an $L \times L$ identity matrix, which amounts to measuring random frequency points at the $i$ th scan point. This reduces the data acquisition time by a factor of $L / M$ for SF-GPRs. Note that $\boldsymbol{\Phi}_{i}$ can be different at each scan point. Using measurements $\beta_{i}$ from $S$ different scan positions, the target space $\boldsymbol{b}$ is constructed with conventional $\mathrm{CS}^{5,7}$ by solving a combined optimization problem as

$$
\hat{\boldsymbol{b}}=\operatorname{argmin}\|\boldsymbol{b}\|_{1} \quad \text { s.t. }\|\boldsymbol{\beta}-\boldsymbol{A} \boldsymbol{b}\|_{2}<\epsilon,
$$

where $\quad \beta=\left[\beta_{1}^{T}, \ldots, \beta_{S}^{T}\right]^{T}, \quad \Psi=\left[\Psi_{1}^{T}, \ldots, \Psi_{S}^{T}\right]^{T}, \quad \Phi=$ $\operatorname{diag}\left\{\Phi_{1}, \ldots, \Phi_{S}\right\}$, and $A=\Phi \Psi$ and $\epsilon$ is a regularization parameter of the problem. Here the selected $S$ scan positions can also be a random subset of the scan positions within the whole synthetic aperture. The optimization problem in Eq. (7) can all minimize convex functionals and global optimal solutions can be obtained through linear programming. Instead of solving Eq. (7), suboptimal greedy algorithms are also used in many applications owing to their lower computational complexity. OMP, ${ }^{23}$ compressive sampling matching pursuit (CoSamp), ${ }^{26}$ and iterative hard/soft thresholding $(\mathrm{IHT})^{27}$ are some of these greedy algorithms. We used OMP as a basic iterative scheme for our proposed offgrid reconstruction technique as detailed in the next section.

\section{Gradient-Based OMP for Off-Grid Targets}

Actual targets can be at any spatial position within the imaging area, but conventional reconstruction techniques such as OMP try to generate a sparse target space image by identifying the grid cells in which the targets are present. OMP method starts with an empty support set $S$, and the most correlated column of $\boldsymbol{A}$ with the current residual is added to the support list, increasing the span of the current support at each iteration. Hence at each new iteration of OMP, a new grid

Table 1 OMP Algorithm.

$\operatorname{OMP}(\boldsymbol{A}, \boldsymbol{\beta}, \epsilon)$ :

Initialization: $S_{0}=\{\}, \boldsymbol{r}_{0}=\boldsymbol{\beta}, \boldsymbol{k}=1$

While loop, repeat until $\|\boldsymbol{r}\|_{2}<\epsilon$

$$
\begin{aligned}
& \lambda_{k}=\operatorname{argmax}_{w}\left|\left\langle\boldsymbol{r}_{k-1} \boldsymbol{A}_{w}\right\rangle\right| \\
& S_{k}=\left[S_{k-1} \lambda_{k}\right] \\
& \boldsymbol{U}=\boldsymbol{A}\left\{:, S_{k}\right\} \alpha_{k}=\boldsymbol{U}^{\dagger} \boldsymbol{\beta} \\
& \boldsymbol{r}_{k}=\boldsymbol{\beta}-\boldsymbol{U}_{\alpha_{k}}
\end{aligned}
$$

Increase $k$ by 1

Output: $\boldsymbol{b}^{*}\left(S_{k}\right)=\boldsymbol{U}^{\dagger} \boldsymbol{\beta}$ 
point is added to the target list. Then, projection of the measurements onto current support is computed to obtain an estimate for that iteration level. This procedure is repeated until the residual norm is less than a given tolerance level of $\epsilon$ or a predetermined sparsity level. The main process of OMP is summarized in Table 1.

In general, a target at position $\boldsymbol{p}_{k}$ may not be exactly on the grid center, but it is at an unknown shift $\Delta \boldsymbol{p}_{k}=$ $\left[\Delta x_{k} ; \Delta y_{k} ; \Delta z_{k} ;\right]$ from the grid center and still in the grid area, where $\left|\delta x_{k}\right|<\Delta_{x} / 2,\left|\delta y_{k}\right|<\Delta_{y} / 2$, and $\left|\delta z_{k}\right|<\Delta_{z} / 2$ with $\Delta_{x}, \Delta_{y}, \Delta_{z}$ defining the grid dimensions in $x, y$, and $z$ axis, respectively. It is expected that the measurements due to this off-grid target will be correlated the most with its own grid center, and OMP procedure will select the dictionary column corresponding to that grid center to represent response from this target. A desired algorithm would be able to find the unknown shift $\Delta \boldsymbol{p}_{k}$ and add the model data for that target point to the support vector list.

OMP procedure in Table 1 calculates a cost function of $\left|\left\langle\boldsymbol{r}_{k-1} \boldsymbol{A}_{w}\right\rangle\right|$ at only grid centers by projecting the combined measurements from all scan points to the combined data dictionary and tries to maximizes this cost. If we had the chance to calculate this cost at every spatial position, then this cost would be defining a continuous surface on the target space and the goal would be to find the peaks of this surface. One example cost-function image over the target space with three off-grid targets with underlying grid centers is shown in Fig. 1.

Although such a continuous cost function peaks at the correct target locations, it is only evaluated at the grid centers. However, if gradients of this cost function can be calculated at a given position, then starting from the grid center a steepest ascent-type algorithm can be used to find the peak of the cost function within the grid. Here we simply propose to use this gradient-based optimization of the cost initially starting from the grid point selected by the OMP algorithm and by taking controlled steps in the direction of the gradient, sequentially updating the target position until a convergence criterion is met. Hence the proposed idea can be summarized as follows: (1) First initialize the target point to the selected grid center from OMP iteration as $\boldsymbol{p}_{k, 0}=\boldsymbol{\pi}_{n}$. (2) Start from $s=1$ until convergence perform updates

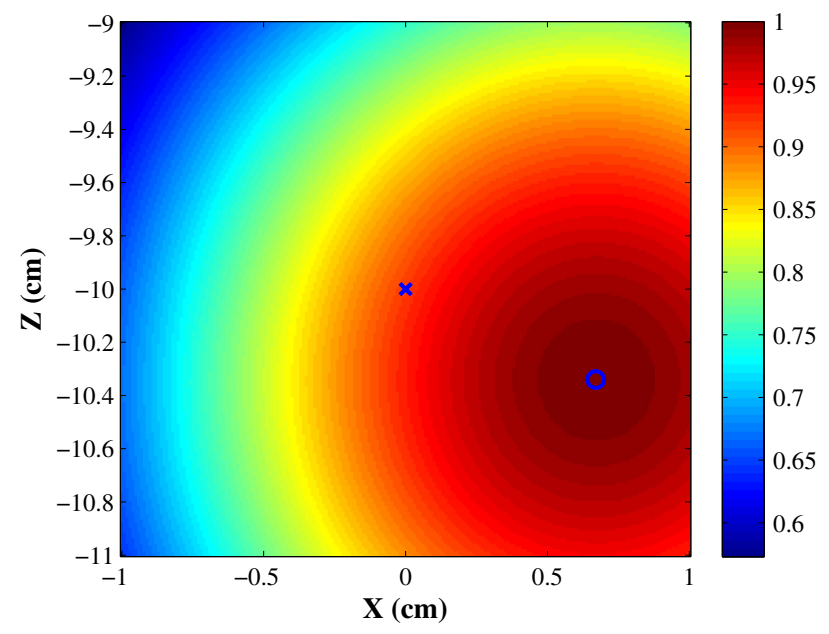

Fig. 1 Evaluation of the orthogonal matching pursuit (OMP) cost over the target space in a single grid. $x$, grid center; $o$, true position of the target.

$$
\boldsymbol{p}_{k, s}=\boldsymbol{p}_{k, s-1}+\mu_{s}\left[\left.\frac{\partial J}{\partial x}\right|_{\boldsymbol{p}_{k, s-1}} ;\left.\frac{\partial J}{\partial y}\right|_{\boldsymbol{p}_{k, s-1}} ;\left.\frac{\partial J}{\partial z}\right|_{\boldsymbol{p}_{k, s-1}}\right] .
$$

(3) Terminate if derivatives are smaller than a threshold where $\boldsymbol{p}_{k, s}$ is the updated target point for iteration $s, \mu_{s}$ is a step-size vector having step sizes in each of $x, y$, and $z$ directions, and

$$
\left.\frac{\partial J}{\partial x}\right|_{\boldsymbol{p}_{k, s-1}}
$$

is the partial derivative of the cost function $J$ with respect to a spatial dimension $x$ evaluated at the point $\boldsymbol{p}_{k, s-1}$. The convergence rate of this steepest ascent-based algorithm is only linear, since the it is applied on a convex quadratic function. This steepest-ascent procedure requires the evaluation of partial derivatives in each direction at the updated target points. The cost function and its derivatives can also be calculated easily using the GPR data model relation. To do so, first define the projection of the measurements to the data model for a target at point $\boldsymbol{p}$ as

$$
C(\boldsymbol{p})=\sum_{i=1}^{S} \boldsymbol{\beta}_{i}^{H} \frac{e^{-j \boldsymbol{\omega}\left[\tau_{i}(p)\right]}}{\sqrt{M}},
$$

where $\boldsymbol{\beta}_{i}^{H}$ is the hermitian transpose of $\boldsymbol{\beta}_{i}$. The cost function calculated by OMP is the absolute of $C(\boldsymbol{p})$ which can be stated as

$$
J(\boldsymbol{p})=\sqrt{C(\boldsymbol{p}) C(\boldsymbol{p})^{H}} .
$$

Hence the partial derivatives with respect to spatial axis can be calculated as

$$
\frac{\partial J}{\partial x}=\frac{1}{2 \sqrt{C(\boldsymbol{p}) C^{H}(\boldsymbol{p})}}\left[\frac{\partial C(\boldsymbol{p})}{\partial x} C^{H}(\boldsymbol{p})+\frac{\partial C^{H}(\boldsymbol{p})}{\partial x} C(\boldsymbol{p})\right] .
$$

Partial derivatives with respect to $y$ and $z$ can be defined similar to Eq. (10). Calculation of Eq. (10) requires the evaluation of the following derivatives:

$$
\begin{gathered}
\frac{\partial C(\boldsymbol{p})}{\partial x}=\sum_{i=1}^{S} \boldsymbol{\beta}_{i}^{H} \frac{e^{-j \omega\left[\tau_{i}(p)\right]}}{\sqrt{M}}(-j \boldsymbol{\omega}) \frac{\partial \tau_{i}(\boldsymbol{p})}{\partial x} \\
\frac{\partial C^{H}(\boldsymbol{p})}{\partial x}=\sum_{i=1}^{S} \boldsymbol{\beta}_{i} \frac{e^{j \omega\left[\tau_{i}(\boldsymbol{p})\right]}}{\sqrt{M}}(j \boldsymbol{\omega}) \frac{\partial \tau_{i}(\boldsymbol{p})}{\partial x} .
\end{gathered}
$$

Note that calculation of the derivatives in Eq. (11) needs simply $C(\boldsymbol{p})$ and $C^{H}(\boldsymbol{p})$ and the partial derivative of the time delay with respect to spatial axes,

$$
\frac{\partial \tau_{i}(\boldsymbol{p})}{\partial x}
$$

which can be easily computed depending on the data acquisition geometry. The step size $\mu_{s}$ can be given a priori, or locally optimal step sizes can be selected with line search at every iteration. The proposed method using the OMP 
Table 2 Gradient Based OMP Algorithm.

$$
\begin{aligned}
& \text { OMP }(\boldsymbol{A}, \boldsymbol{\beta}, \epsilon) \text { : } \\
& \text { Initialization: } \boldsymbol{S}_{0}=\{\}, \boldsymbol{U}_{0}=\{\}, \boldsymbol{r}_{0}=\boldsymbol{\beta}, k=1 \\
& \text { While loop, repeat until }\|\boldsymbol{r}\|_{2}<\epsilon \\
& \lambda_{k}=\operatorname{argmax}_{w}\left|\left\langle\boldsymbol{r}_{k-1} \boldsymbol{A}_{w}\right\rangle\right| \\
& \text { Run Gradient Iterations Until Terminated: } \\
& \quad \text { Initialize } \boldsymbol{s}=1, \mu_{s-1}=[\Delta \boldsymbol{x}, \Delta y, \Delta z] / 10, \boldsymbol{p}_{k, s-1}=\boldsymbol{\pi}_{\lambda_{k}} \\
& \quad \text { Evaluate } \boldsymbol{G}=\left.\left[\frac{\partial J}{\partial x}, \frac{\partial J}{\partial y}, \frac{\partial J}{\partial z}\right]\right|_{p_{k, s-1}} \\
& \quad \text { Take a step } \boldsymbol{p}_{k, s}=\boldsymbol{p}_{k, s-1}+\mu_{s-1} \boldsymbol{G} \\
& \quad \boldsymbol{s}=\boldsymbol{s}+1 \text { and } \mu_{s}=c * \mu_{s-1} \text { with } c<1 \\
& \quad \text { Output: } \boldsymbol{p}=\boldsymbol{p}_{k, s} \\
& S_{k}=\left[\boldsymbol{S}_{k-1} \boldsymbol{p}\right] \\
& \boldsymbol{U}_{k}=\left\{\boldsymbol{U}_{k-1} \exp -j \boldsymbol{\omega}[\tau(\boldsymbol{p})]\right\} \alpha_{k}=\boldsymbol{U} \dagger \boldsymbol{\beta} \\
& \boldsymbol{r}_{k}=\boldsymbol{\beta}-\boldsymbol{U} \alpha_{k}
\end{aligned}
$$

Increase $k$ by 1

Output: $\left\{\alpha_{k}, S_{k}\right\}$

framework and the detailed gradient-based iterations is summarized in Table 2.

\section{Numerical Simulations and Results}

In this section, performance of the proposed algorithm is analyzed for sparse GPR imaging in the case of targets that can be arbitrarily located within the target space. Hence the targets can be off the grid. First, an example GPR scenario is simulated. A stepped-frequency GPR scanning a bandwidth from $500 \mathrm{MHz}$ to $5.5 \mathrm{GHz}$ with $20 \mathrm{-Mhz}$ step sizes is assumed. A two-dimensional (2-D) target space covering between $[-0.5,0.5] \mathrm{m}$ in $x$ direction and $[0,-1] \mathrm{m}$ in depth $(z)$ dimensions containing three randomly placed point targets is generated, and the corresponding SF-GPR response of the target region is simulated. The target region is scanned at 51 uniformly spaced scan points with $2-\mathrm{cm}$ increments, and at each scan point $20 \%$ of the total frequency steps are randomly measured. To generate the data dictionary, the specified target domain is discretized with 5-cm grid size in both $x$ and $z$ dimensions. The target space image is inverted using both OMP and the proposed gradient-based OMP (GB-OMP) techniques. Both techniques use the same measurements, data dictionary, and termination criterion, which is selected for this example as $\epsilon=0.05\|\boldsymbol{\beta}\|_{2}$.

Figure 2(a) shows the correct positions of the point targets. Solution of the proposed technique and OMP are given in Fig. 2(b) and 2(c), respectively. It can be seen that whereas OMP has higher peaks around the true target regions, it is not a sparse image and it has selected too many grid points to satisfy the data contraint. However, the proposed method directly finds three off-grid targets at their nearly correct positions. Figure 3(a) shows the same simulation result as a 2-D image with underlying grids and their centers. The true locations of the targets are shown with a circle, where the reconstructed target locations (proposed technique) are shown with a plus sign. It can be seen that GB-OMP found the target parameters very close to the correct values. Figure 3(b) shows the gradient-based steps taken for one of the targets starting from the grid center until the gradient iterations are terminated. It can be seen that with decreasing step sizes, the algorithm converges to the correct target location.

One important point is to understand the effect of the grid sizes on the compared reconstruction technique. Because grid size determines the size of both the reconstruction problem and the data model dictionary, being able perform successfully at bigger grid sizes decreases the computation and memory requirements. Thus, a comparison between OMP and the GB-OMP techniques for the same off-grid target space with four-point targets is done with grid spacings of 5,10 , and $20 \mathrm{~cm}$. In all cases, the same termination criterion is used. The reconstructed target spaces for both techniques are shown in Fig. 4. The proposed technique finds exactly four targets at their nearly correct locations when the grid size is 5 or $10 \mathrm{~cm}$. When the grid size is increased to $20 \mathrm{~cm}$, proposed method finds two of the four targets correctly but fail to find other targets and generates false targets at wrong positions. OMP in all cases creates a nonsparse images and suffers from the increased grid size more than the proposed technique. Although higher peaks around the

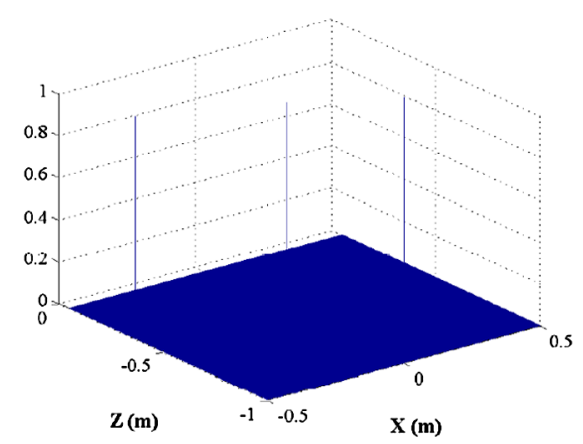

(a)

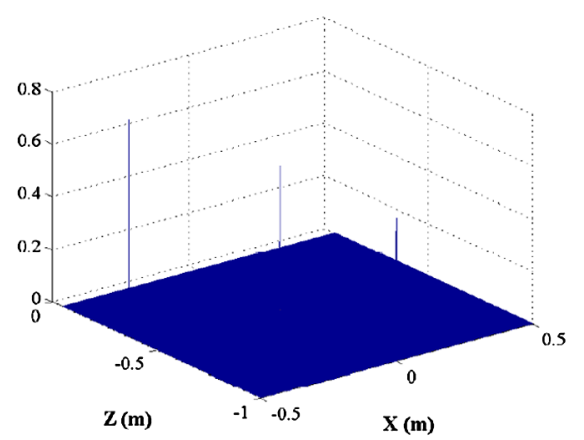

(b)

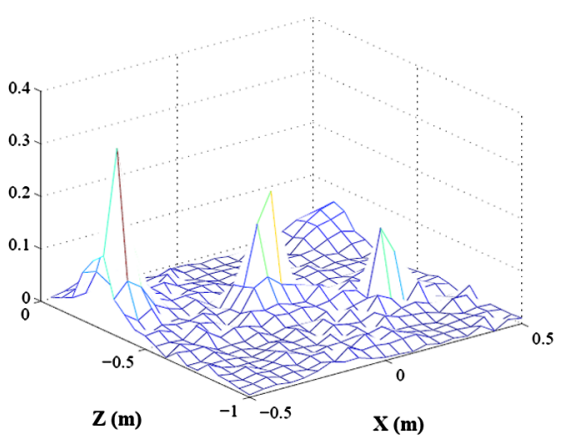

(c)

Fig. 2 (a) True ground-penetrating radar (GPR) target space with $K=3$ off-the-grid targets. (b) Gradient-based (GB)-OMP reconstruction result. (c) OMP reconstruction result. 


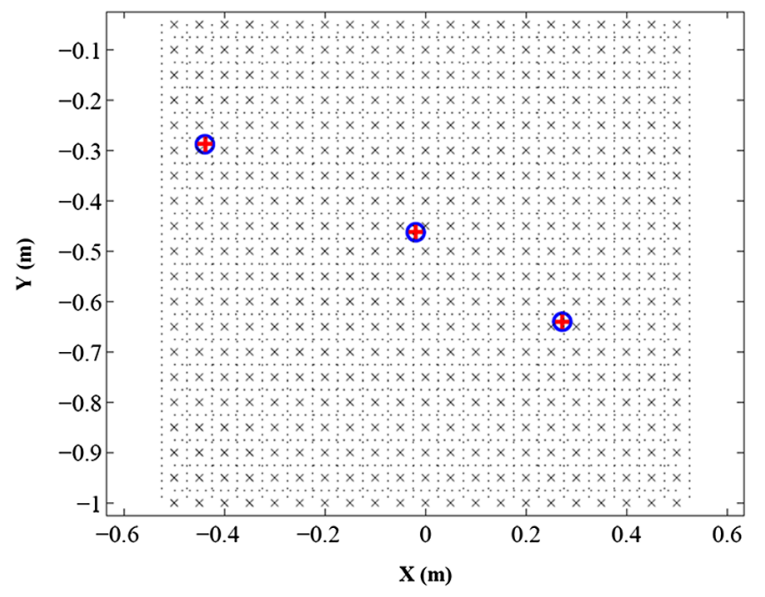

(a)

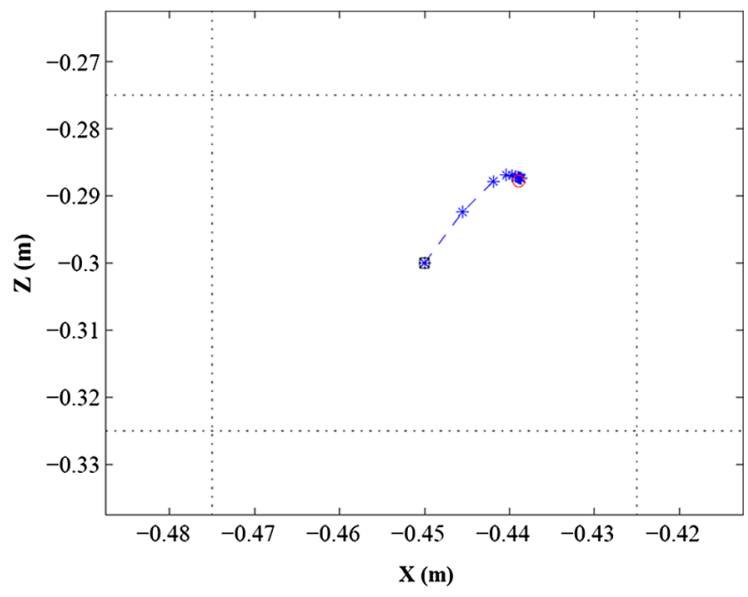

(b)

Fig. 3 (a) Reconstructed target positions with the proposed technique with the underlying grids. $\bigcirc$, correct target parameters; +, parameters found by the proposed method; $x$, grid centers. (b) Gradient-based steps taken within the proposed algorithm at one of the target grids.

target region are obtained for the 5-cm grid size, OMP starts to lose the targets with increasing grid sizes.

The average performance and robustness of the proposed technique has also been analyzed in terms of parameters such as sparsity level and signal-to-noise ratio (SNR). Methods like OMP tend to generate nonsparse reconstructions, since they cannot represent the data with the dictionary columns generated from the grid centers sparsely. To test the performance of the proposed technique on sparsity level of the reconstructed GPR image, GB-OMP method is applied to cases with varying levels of correct sparsity levels changing from 1 to 20 . For each sparsity level, point targets are randomly placed in the target space, and the tested sparsity level and reconstruction is done with both OMP and the GB-OMP techniques. An underlying grid of $5 \mathrm{~cm}$ in both dimensions is taken, and the corresponding data dictionary is used. Both techniques are terminated with the same criterion, and the sparsity levels of the reconstructions are observed. This simulation was repeated 20 times for each sparsity level, with independent and random GPR target space selections. The average level of estimated sparsity versus the true sparsity level for the GB-OMP technique is shown in Fig. 5(a). OMP selected all grid indexes, which is 420 for the grid size used, for all the tested sparsity levels

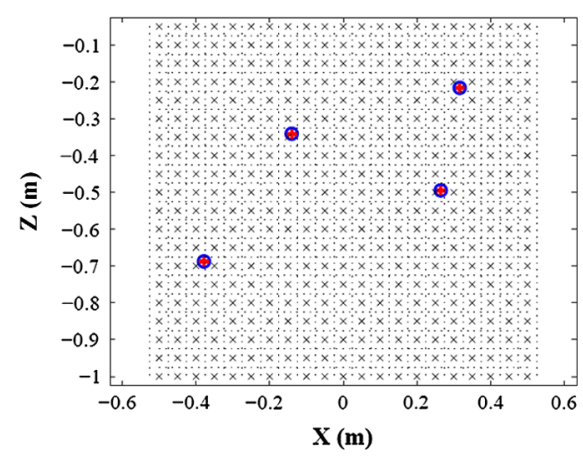

(a)

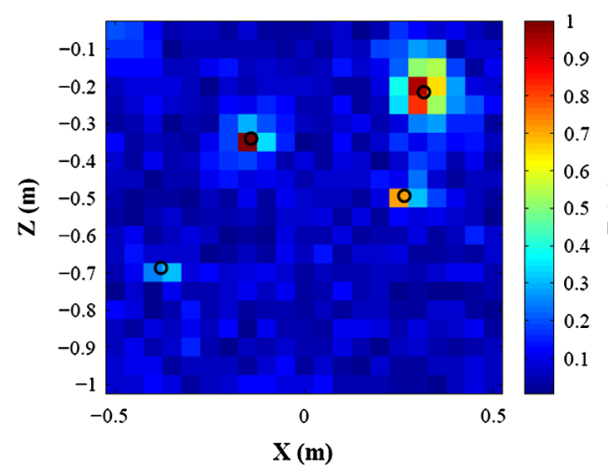

(d)

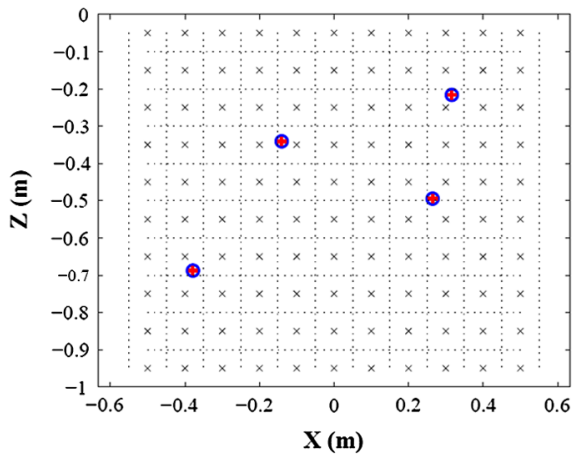

(b)

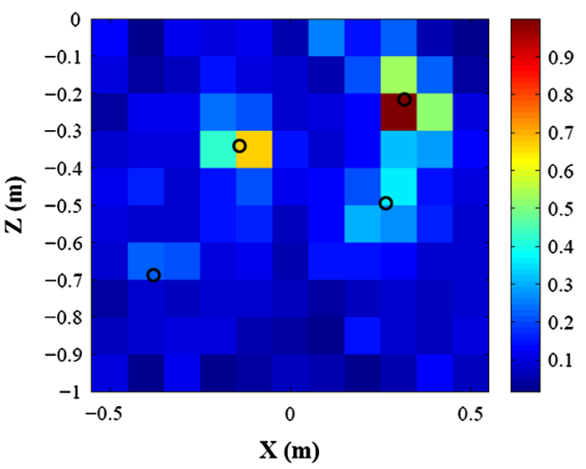

(e)

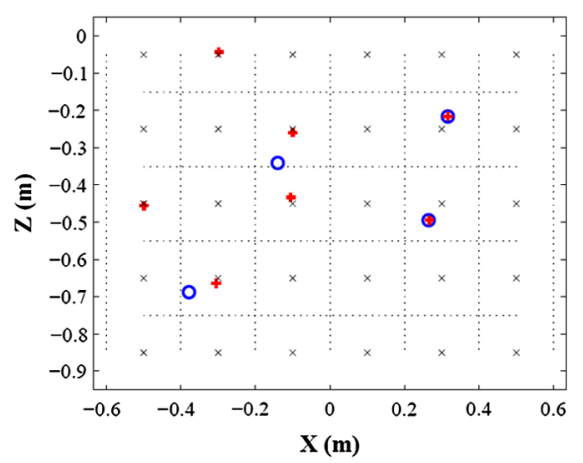

(c)

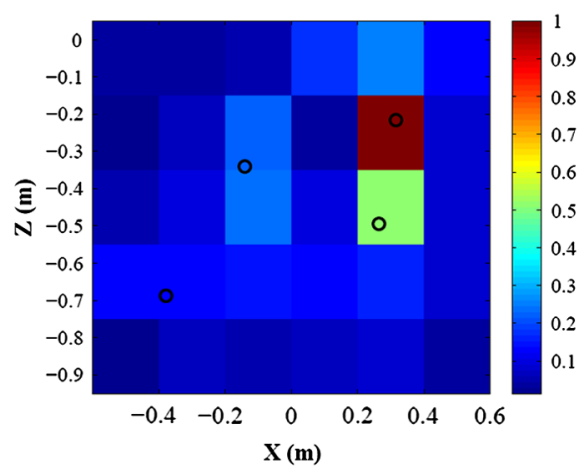

(f)

Fig. 4 GB-OMP results with grid sizes of $5 \mathrm{~cm}$ (a) $10 \mathrm{~cm}$ (b) and $20 \mathrm{~cm}$ (c) Reconstructed OMP images with grid sizes of $5 \mathrm{~cm}$ (d) $10 \mathrm{~cm}($ e) and $20 \mathrm{~cm}(\mathrm{f})$. 


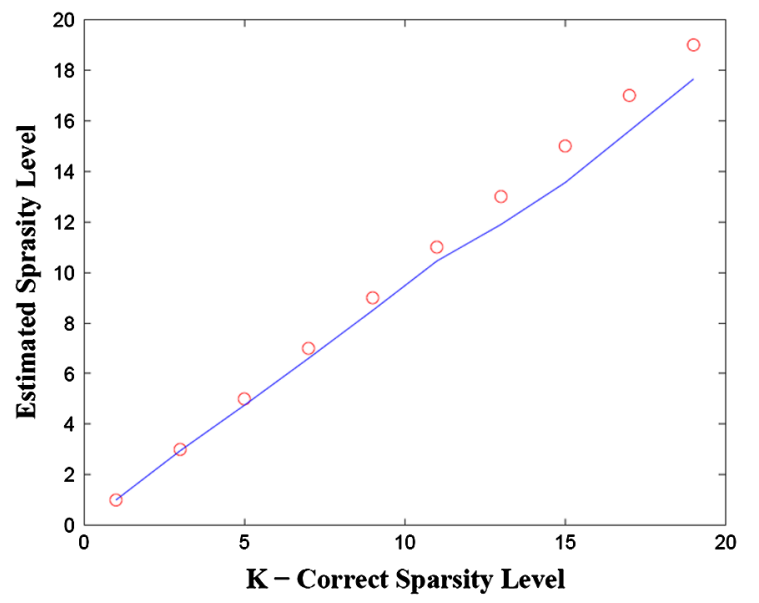

(a)

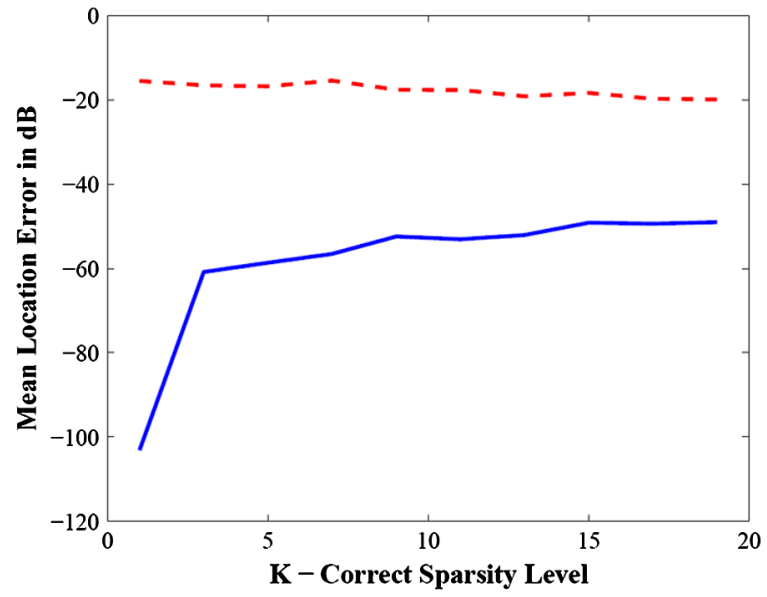

(b)

Fig. 5 (a) Estimated sparsity level of the GB-OMP versus the correct sparsity of the target space. (b) Average location error in dB versus the sparsity level of the scene for OMP and GB-OMP methods.

to satisfy the termination constraint. On the other hand, GBOMP reconstructs the target space with the correct sparsity level until the sparsity level of the scene is about 13 for the simulation parameters. For scenes with higher numbers of targets, it is observed that GB-OMP generates slightly fewer targets then the true sparsity level.

Another important metric is the average location error in the detected targets. For this purpose, target spaces with varying numbers of sparsity from 1 to 20 are generated, and for each sparsity level, the absolute distance with the true and estimated target locations is calculated for both OMP and GB-OMP techniques. Because both techniques may generate target spaces with more than $K$ targets, the best $K$ representation (meaning locations of the $K$ targets having the highest reconstruction values) are compared for both methods. This simulation was repeated 20 times with independent random target space generations, and the average of the location errors is calculated in decibels since OMP error is comparably higher than GB-OMP. Figure 5(b) shows this average location error for varying sparsity levels for both OMP and GB-OMP techniques. It can be observed that the GB-OMP method could estimate the target locations with much lower errors compared to OMP. The parameter error

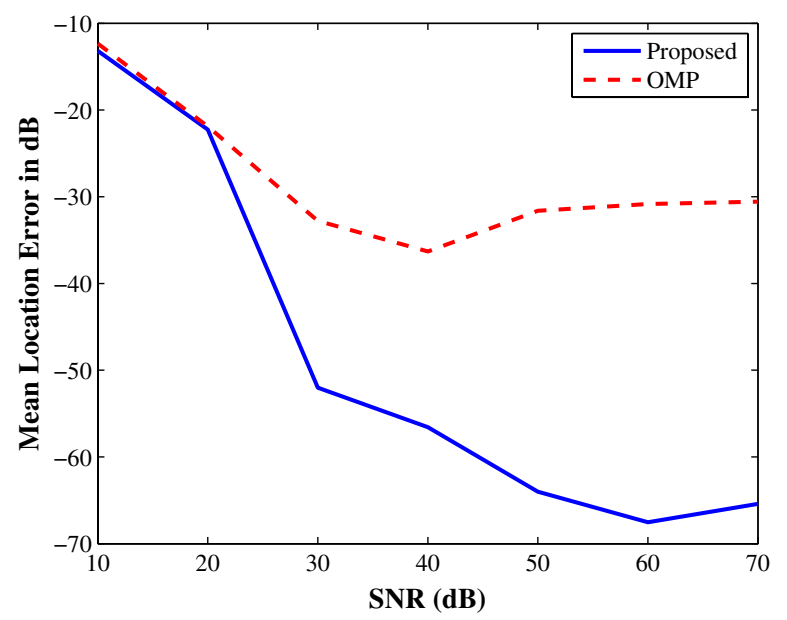

Fig. 6 Average location error in $\mathrm{dB}$ versus varying levels of SNR. for OMP seems to be flat for varying sparsity levels, since OMP only selects the grid centers. Error for GB-OMP is much lower compared to OMP, and the error increases slightly with the increasing level of target space sparsity.

To investigate noise performance of the GB-OMP method in varying levels of noise power, it was tested under SNR levels from 10 to $70 \mathrm{~dB}$. For each SNR level, a random target space with a sparsity level of $K=3$ is generated. Additive white Gaussian noise with corresponding noise power is added to the simulated received signal. As a performance metric, the average absolute location errors are calculated for the detected targets for the compared methods. This simulation was repeated 20 times with independent target space and noise generations. Figure 6 shows the average location errors for the tested SNR levels. It is observed that the average errors are significantly lower than the OMP parameter errors for SNR levels $>20 \mathrm{~dB}$.

An important discussion on this topic is the effect of offgrid targets to the receiver operating characteristics of a system. From Eq. (1), a mismatch in the sparsity basis results in an increase in the error bound by an amount of $(N-k) \beta\|\theta\|_{2}$, which shows that any probability-ofdetection value will be achieved with a higher probabilityof-false-alarm ratio when a basis mismatch occurs and conventional reconstruction techniques such as basis pursuit or OMP are used. Although we have not proven theoretically here, our proposed method removes the leakage due to off-grid targets to the other grid cells, hence expectedly decreasing false alarms due to the off-grid phenomenon.

\section{Conclusions}

Although CS brings important advantages to GPR imaging, because of the off-the-grid target problem, CS techniques had significant challenges in this application. A new gradient-based iterative algorithm that can reconstruct the target under off-grid targets is proposed in this paper. The proposed technique calculates gradient base steps toward maximizing the projection function cost for each selected grid. Simulations show that sparse off-grid GPR imaging can be successfully done with the proposed technique. Compared to the standard OMP technique, the proposed method 
provides significantly lower localization errors for a wide range of sparsity levels and SNRs.

\section{Acknowledgments}

This work was supported by TUBITAK within Career Program Grant Compressive Remote Sensing and Imaging, project number 109E280, and within FP7 Marie Curie IRG Grant Compressive Data Acquisition and Processing Techniques for Sensing Applications, project number PIRG04-GA-2008-239506.

\section{References}

1. H. M. Jol, Ground Penetrating Radar Theory and Applications, Elsevier, New York (2009).

2. M. G. Amin, Through-the-Wall Radar Imaging, CRC Press, Boca Raton, Florida (2010).

3. D. Donoho, "Compressed sensing," IEEE Trans. Inform. Theor. 52(4), 1289-1306 (2006).

4. E. Candes, J. Romberg, and T. Tao, "Robust uncertanity principles: exact signal reconstruction from highly incomplete frequency information," IEEE Trans. Inform. Theor. 52(2), 489-509 (2006).

5. E. Candès, J. Romberg, and T. Tao, "Stable signal recovery from incomplete and inaccurate measurements," Comm. Pure Appl. Math. 59(8), 1207-1223 (2006).

6. J. Haupt and R. Nowak, "Signal reconstruction from noisy random projections," IEEE Trans. Inform. Theor. 52(9), 4036-4048 (2006).

7. D. Donoho, M. Elad, and V. Temlyakov, "Stable recovery of sparse overcomplete representations in the presence of noise," IEEE Trans. Inform. Theor. 52(1), 6-18 (2006).

8. R. Baraniuk and P. Steeghs, "Compressive radar imaging," in Proc. IEEE Radar Conf., pp. 128-133, IEEE, Boston, Massachusetts (2007).

9. A. C. Gurbuz, J. H. McClellan, and W. R. Scott Jr., "Compressive sensing for GPR imaging," in Proc. IEEE Conf. Record of the 41st Asilomar Conf. on Signals, Systems and Computers, pp. 2223-2227, IEEE, Pacific Grove, California (2007).

10. A. C. Gurbuz, J. H. McClellan, and W. R. Scott Jr., "A compressive sensing data acquisition and imaging method for stepped frequency GPRs," IEEE Trans. Signal Process. 57(7), 2640-2650 (2009).

11. A. C. Gurbuz, J. H. McClellan, and W. R. Scott Jr., "Compressive sensing for subsurface imaging using ground penetrating radars," Signal Process. 89(10), 1959-1972 (2009).

12. Y.-S. Yoon and M. G. Amin, "Imaging of behind the wall targets using wideband beamforming with compressive sensing," in Proc. IEEE 15th Workshop on Statistical Signal Process., pp. 93-96, IEEE, Cardiff, Wales, United Kingdom (2009)

13. A. B. Suksmono et al., "Compressive stepped-frequency continuouswave ground-penetrating radar," IEEE Geosci. Remote Sens. Lett. 7(4), 665-669 (2010).

14. F. Soldovieri et al., "Sparse reconstruction from GPR data with applications to rebar detection," IEEE Trans. Instrum. Meas. 60(3), 10701079 (2011).

15. E. Lagunas et al., "Joint wall mitigation and compressive sensing for indoor image reconstruction," IEEE Trans. Geosci. Remote Sens. 51(2), 891-906 (2013).

16. Q. Huang et al., "UWB through-wall imaging based on compressive sensing," IEEE Trans. Geosci. Remote Sens. 48(3), 1408-1415 (2010).

17. R. Baraniuk et al., "A simple proof of the restricted isometry property for random matrices," Constr. Approx. 28(3), 253-263 (2008).

18. M. A. Tuncer and A. C. Gurbuz, "Analysis of unknown velocity and target off the grid problems in compressive sensing based subsurface imaging," in Proc. IEEE Int. Conf. on Acoustics, Speech and Signal Process., pp. 2880-2883, IEEE, Prague, Czech Republic (2011).

19. J. H. Ender, "On compressive sensing applied to radar," Signal Process. 90(5), 1402-1414 (2010).

20. M. Herman and T. Strohmer, "General deviants: an analysis of perturbations in compressed sensing," IEEE J. Sel. Top. Signal Process. 4(2), 342-349 (2010).
21. Y. Chi et al., "Sensitivity of basis mismatch to compressed sensing," IEEE Trans. Signal Process. 59(5), 2182-2195 (2011).

22. D. H. Chae, P. Sadeghi, and R. A. Kennedy, "Effects of basis-mismatch in compressive sampling of continuous sinusoidal signals," in Proc. IEEE 2nd Int. Conf. on Future Comput. and Commun., Vol. 2, pp. 739-743, IEEE, Wuhan, China (2010).

23. J. Tropp and A. Gilbert, "Signal recovery from random measurements via orthogonal matching pursuit," IEEE Trans. Inform. Theor. 53(12), 4655-4666 (2007).

24. M. A. C. Tuncer and A. C. Gurbuz, "Analysis of orthogonal matching pursuit based subsurface imaging for compressive ground penetrating radars," Turk. J. Elect. Eng. Comp. Sci. 20(6), 979-989 (2012).

25. D. Daniels, Ground Penetrating Radar, 2nd ed., Institution of Electrical Engineers (IEE), London (2004).

26. D. Needell and J. A. Tropp, "Cosamp: iterative signal recovery from incomplete and inaccurate samples," Appl. Comp. Harmonic Anal. 26(3), 301-321 (2009).

27. T. Blumensath and M. E. Davies, "Iterative hard thresholding for compressed sensing," Appl. Comput. Harm. Anal. 27(3), 265-274 (2009).

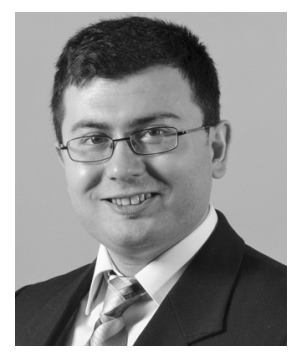

Ali Cafer Gurbuz received the BS degree from Bilkent University, Ankara, Turkey, in 2003 in electrical and electronics engineering, and the $\mathrm{MS}$ and $\mathrm{PhD}$ degrees from the Georgia Institute of Technology, Atlanta, in 2005 and 2008, both in electrical and computer engineering. From 2003 to 2008, he participated in multimodal landmine detection system research as a graduate research assistant and from 2008 to 2009 , as postdoctoral fellow, all with Georgia Tech. He is currently an assistant professor with TOBB University of Economics and Technology, Ankara, Turkey, with the Department of Electrical and Electronics Engineering. His research interests include compressive sensing applications, ground penetrating radar, array signal processing, remote sensing, and imaging.

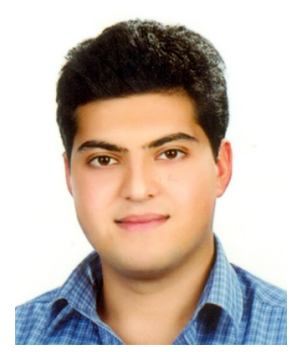

Oguzhan Teke received the BSc degree in electrical and electronics engineering from Bilkent University, Ankara, Turkey, in 2012. $\mathrm{He}$ is currently working toward the MS degree under the supervision of Prof. Orhan Arikan in the Department of Electrical and Electronics Engineering, Bilkent University. His research interests are in applied linear algebra and numerical analysis, inverse problems, and compressed sensing.

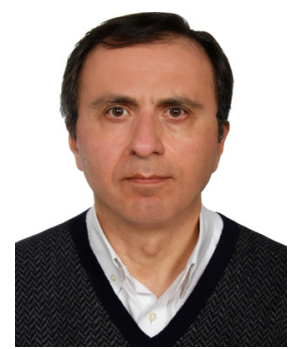

Orhan Arikan received his BSc degree in electrical and electronics engineering from the Middle East Technical University, Ankara, Turkey. He received both his MS and $\mathrm{PhD}$ degrees in electrical and computer engineering from the University of Illinois, Urbana-Champaign, in 1988 and 1990, respectively. Following his graduate studies, he was employed as a research scientist at Schlumberger-Doll Research Center, Ridgefield, CT. In 1993, he joined the Electrical and Electronics Engineering Department of Bilkent University, Ankara, Turkey. Since 2011, he is serving as the department chairman. His current research interests include statistical signal processing, time-frequency analysis, and remote sensing. He has served as chairman of IEEE Signal Processing Society Turkey Chapter and president of IEEE Turkey Section. 